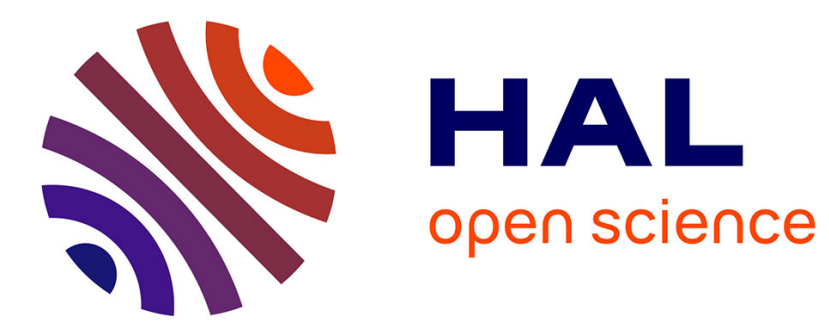

\title{
Magnetic anisotropy and domain duplication in transport properties of tunnel junctions
}

\author{
M. Hehn, O. Lenoble, D. Lacour, Alain Schuhl
}

\section{To cite this version:}

M. Hehn, O. Lenoble, D. Lacour, Alain Schuhl. Magnetic anisotropy and domain duplication in transport properties of tunnel junctions. Physical Review B: Condensed Matter (1978-1997), 2000, 62 (17), pp.11344-11346. 10.1103/PhysRevB.62.11344 . hal-02954383

\section{HAL Id: hal-02954383 \\ https://hal.science/hal-02954383}

Submitted on 1 Oct 2020

HAL is a multi-disciplinary open access archive for the deposit and dissemination of scientific research documents, whether they are published or not. The documents may come from teaching and research institutions in France or abroad, or from public or private research centers.
L'archive ouverte pluridisciplinaire HAL, est destinée au dépôt et à la diffusion de documents scientifiques de niveau recherche, publiés ou non, émanant des établissements d'enseignement et de recherche français ou étrangers, des laboratoires publics ou privés. 


\section{Magnetic anisotropy and domain duplication in transport properties of tunnel junctions}

Article in Physical Review B · November 2000

DOl: 10.1103/PhysRevB.62.11344

CITATIONS

10

4 authors, including:

Michel Hehn

University of Lorraine

278 PUBLICATIONS 6,092 ATATIONS

SEE PROFILE

Alain Schuhl

(3) UniversityJ oseph Fourier - Grenoble 1

142 PUBLICATIONS 6,044 ATATIONS

SEE PROFILE

Some of the authors of this publication are also working on these related projects:

SAWSensors for Magnetic Field Detection View project

Artificial spin ice Vew project
READS

17

Daniel Lacour

French National Centre for Scientific Research

149 PUBLICATIONS 2,156 ATATIONS

SEE PROFILE 


\title{
Magnetic anisotropy and domain duplication in transport properties of tunnel junctions
}

\author{
M. Hehn, O. Lenoble, D. Lacour, and A. Schuhl \\ Laboratoire de Physique des Matériaux, UMR CNRS 7556, Université H. Poincaré - Nancy 1, \\ Boite Postale 239, 54506 Vandoeuvre Lès Nancy Cedex, France
}

(Received 27 April 2000)

\begin{abstract}
A tunnel magnetoresistance signal is used for probing the relative orientations of the local magnetization in the two magnetic electrodes of a tunnel junction. Minor magnetoresistance loops are studied as a function of the return field in order to change the domain configuration of the hard layer. Domain structure duplication of the hard layer template into the soft layer is observed. This effect is shown to be driven by the magnetic anisotropy of the hard layer. Reducing this anisotropy leads to domain duplication but with effects nondiscernible in the tunnel magnetoresistance (TMR) signal. However, a signature of the $360^{\circ}$ domain walls is then observed on the TMR signal.
\end{abstract}

Typical magnetic tunnel junctions make use of two magnetic layers with different coercivities, respectively called hard and soft, separated by a 1- to 2-nm-thick insulating layer. With such thin spacer, magnetic coupling between the two electrodes usually takes place. This coupling can affect dramatically the behavior of the tunnel junction based devices. So rapidly after the emergence of magnetic tunnel junctions, ${ }^{1}$ indirect coupling has been intensively studied. Indeed, magnetostatic interactions induced by domain walls are known to play an important role in the reversal properties of the ferromagnetic electrodes ${ }^{2,3}$ and, consequently, in the tunnel magnetoresistance (TMR) signal. ${ }^{4}$ Recently, it has been shown that domain walls sweeping through the soft magnetic layer during its reversal generates an important stray field which leads to a progressive demagnetization of the hard magnetic layer. ${ }^{5}$ In the same way, the TMR signal has been used to study the magnetism of the electrodes and their mutual influence during magnetization reversal. ${ }^{6,7}$

We have recently reported on the induced domain structure in the soft layer duplicated from the hard magnetic template by a ferromagnetic-type coupling. ${ }^{6}$ For the TMR point of view, the junction appears then in a fully parallel state while domains with opposed magnetization are still present in both layers. Uniaxial anisotropy in the hard magnetic layer induces a domain structure with magnetization oriented along the easy axis. The stray field of each domain can either increase or decrease the effective field seen by the soft layer, depending on its orientation with respect to the applied field. So this nonhomogeneous field can induce a domain structure in the soft layer if the energy needed to create domain walls is lower than the coupling energy. While magnetic coupling has been shown to be one key parameter, here we shed light on the role of anisotropy in the occurrence of the domain duplication phenomena.

For this purpose, we have prepared two different sets of samples differing only through the properties of the hard magnetic electrode. In the first set $S 1$, the hard electrode is magnetically anisotropic, whereas in the set $S 2$, the hard layer is magnetically isotropic. Both soft magnetic electrodes $\left[\mathrm{Co}(10 \mathrm{~nm})\right.$, denoted by $\left.\mathrm{Co}^{\text {se }}\right]$ and tunnel barriers $[\mathrm{Al}(1.2$ $\mathrm{nm})$ oxidized $30 \mathrm{~s}$ ] are identical for all the samples. Details of the junction fabrication (oxidation process to make the
AlOx tunnel barrier, geometry for $\mathrm{CCP}$ measurements, etc.) can be found elsewhere. ${ }^{6}$ We take advantage of previous studies to tailor the magnetic properties of the hard layers. ${ }^{8,9}$ When Co is deposited at low Ar pressure (5 $\times 10^{-3}$ mbar), the magnetization reversal is sharp with nucleation and propagation of domain walls. ${ }^{6}$ In addition, this low-pressure process leads to the appearance of an anisotropy axis due to the geometry of the deposition. When the $\mathrm{Ar}$ pressure increases up to an optimum, equal to 1.5 $\times 10^{-2}$ mbar, the grain size and the coercive field increase up to a maximum. In this case, the magnetization behavior is consistent with a reversal occurring through the appearance of ripple domain structures. ${ }^{10}$ More important, the magnetic anisotropy stabilized at low pressure completely disappears. Then, the hard layer of samples $S 2$ is a single Co*(20 nm) layer where $\mathrm{Co}^{*}$ is relative to high Ar pressure. Magnetization reversal occurs then only by rotation and the magnetization cycle is fully isotropic in the plane of the sample. In contrast, for the sample $S 1$, we grew an anisotropic $\mathrm{Co}(5 \mathrm{~nm}) / \mathrm{Co}^{*}(20 \mathrm{~nm})$ hard layer, denoted by $\mathrm{Co} / \mathrm{Co}^{*}$, where $\mathrm{Co}$ is relative to low Ar pressure. Its magnetic behavior is similar to the hard magnetic layer used in our previous study ${ }^{6}$ made with a $\mathrm{Co}(10 \mathrm{~nm})$ layer oxidized $1 \mathrm{mn}$ we denote by $\mathrm{Co} / \mathrm{CoO}$. These two last hard layers $\left(\mathrm{Co} / \mathrm{Co}^{*}\right.$ and $\mathrm{Co} / \mathrm{CoOx}$ ), have the same magnetic structure: the soft anisotropic Co layer is exchange biased by a layer composed of low interacting grains for which reversal occurs with the appearance of ripple-type domain structure. As a result, the domain propagation is hindered in the case of $\mathrm{Co} / \mathrm{Co}^{*}$ or nearly suppressed in the case of $\mathrm{Co} / \mathrm{CoOx}$. An important point concerns the anisotropy of the initial soft Co layer which remains in both cases.

Complete (solid line) and minor (-○-,- - - ) TMR loops are shown in Fig. 1 (respectively, Fig. 2) for typical sample of the set $S 1$ (respectively, $S 2$ ). After saturation at $850 \mathrm{Oe}$, the applied field along the easy axis of the soft $\mathrm{Co}^{\text {se }}$ layer is decreased down to $H_{\text {rev }}$ and reversed in the positive field direction. For the set $S 1$, the sample shows a similar behavior as observed previously with a $\mathrm{Co} / \mathrm{CoOx}$ hard layer (Fig. 1) i.e., the duplication of the domain configuration of the hard magnetic layer in the soft layer for applied field between $H_{1}$ and $H_{2}$. In this case, sharp magnetization rever- 


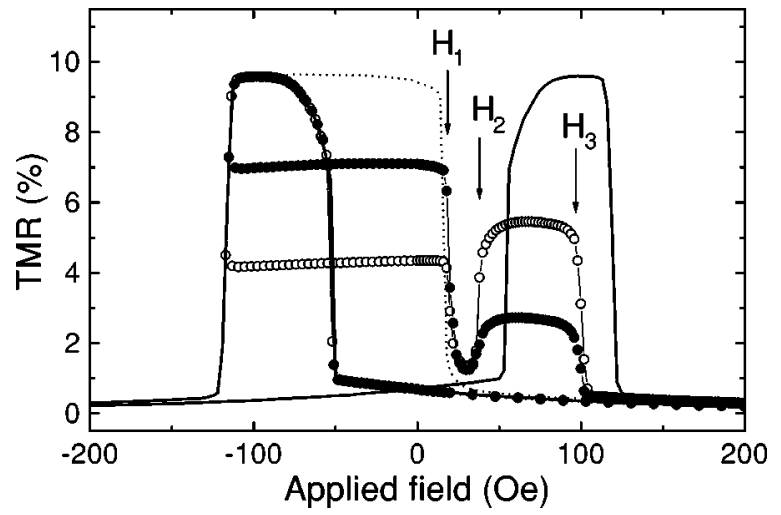

FIG. 1. Complete (-) and three minor tunnel magnetoresistance loops measured on a $\mathrm{Co}^{\text {se }} / \mathrm{Al}(1.2 \mathrm{~nm}$, ox $30 \mathrm{~s}) / \mathrm{Co} / \mathrm{Co}^{*}$ tunnel junction with $200 \mu \mathrm{m}$ lateral size. The three different minor cycles have been obtained with different $H_{\text {rev }}$ values at which the applied field is reversed. By reversing the negative applied field at some $H_{r e v}$ on the minor loop, three jumps with different signs appear at some fields $H_{1}, H_{2}$, and $H_{3}$. The minor loop measured when the applied field is reversed in the positive field direction just after the $\mathrm{Co}^{\text {se }}$ switching (dotted line) is shifted by a bias field of - 17 Oe corresponding to a ferromagnetic coupling with the hard layer.

sal of the hard layer by nucleation and propagation of walls generates regions with the main magnetization oriented in the negative direction which coexist with regions with the main magnetization oriented in the positive field direction. Indeed both directions are parallel (almost) to the easy axis. As $H$ is reversed from $H_{\text {rev }}$ towards the positive fields, regions of the $\mathrm{Co}^{\text {se }}$ (soft layer) which are located over domains in the $\mathrm{Co} / \mathrm{Co}^{*}$ layer with the main magnetization oriented in the positive field direction experience a local field equal to $H+H_{f}$, where $H_{f}$ represents the ferromagnetic field coupling between the two electrodes. Therefore, these regions will rotate first and, after the reversal at $H_{1}$, the two mag-

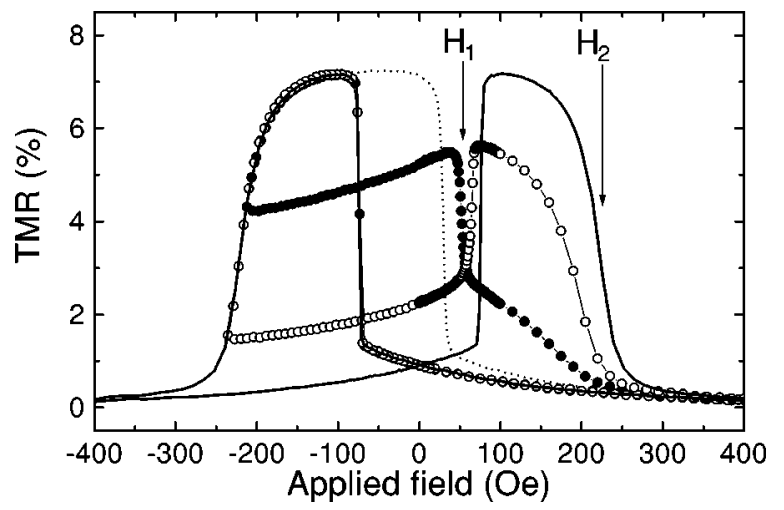

FIG. 2. Complete (-) and three minor tunnel magnetoresistance loops measured on a $\mathrm{Co}^{s e} / \mathrm{Al}(1.2 \mathrm{~nm}$, ox $30 \mathrm{~s}) / \mathrm{Co}^{*}$ tunnel junction with $200 \mu \mathrm{m}$ lateral size. The three different minor cycles have been obtained with different $H_{r e v}$ values at which the applied field is reversed. By reversing the negative applied field at some $H_{\text {rev }}$ on the minor loop, the conventional two jumps attributed to the reversal of the soft and the hard layer appear at some fields $H_{1}$ and $\mathrm{H}_{2}$. The amplitude and the sign of the soft layer reversal jump depend on the percentage of interface reversed magnetization in the hard magnetic layer. netic electrodes hold the same domain structure. Apart from the magnetization inside the walls, locally in both layers, magnetizations are parallel. Then, the junction resistance decreases and is nearly minimal (Fig. 1). A further increase of $H$ leads to the reversal of regions of the $\mathrm{Co}^{\text {se }}$ which are located over domains in the hard layer with the main magnetization oriented in the negative field direction and experience a local field equal to $H-H_{f}$. After this reversal at $H_{2}$, the $\mathrm{Co}^{s e}$ is in a single-domain state while the domain structure remains in the hard layer. Locally in both layers, magnetizations are either parallel or antiparallel and this last magnetic configuration contributes to the increase of the junction resistance. A further increase of $H$ leads finally to the saturation of the hard layer magnetization at $H_{3}$. Here, magnetizations are saturated and parallel. Therefore, resistance decreases and reaches its value at saturation.

With an isotropic hard electrode as for the $S 2$ samples, this signature of the duplication does not appear in the TMR loops (Fig. 2). Indeed, the isotropy of the hard electrode of set $S 2$ leads to a different domain structure. Magnetization reversal of the $\mathrm{Co}^{*}$ layer occurs only by rotation and the magnetization cycle is fully isotropic in plane. When the value of the field applied along the easy axis of the soft $\mathrm{Co}^{\text {se }}$ electrode is reduced from positive saturation to zero, magnetization rotates continuously in the hard $\mathrm{Co}^{*}$ single layer in a reversible process. On a microscopic scale, the sense of rotation is defined by the angle between the local effective magnetocrystalline anisotropy and the external applied field. The magnetic moments are then aligned within an angle bisected by the direction of the applied field. These small angular rotations give rise to an increase of the TMR signal before the $\mathrm{Co}^{\text {se }}$ switching. Here the loop corresponding to the reversal of the $\mathrm{Co}^{\text {se }}$ (dotted line) is shifted by a bias field of -19 Oe corresponding to a ferromagnetic coupling with the hard layer very close to the value observed in set $S 1$ (see caption of Fig. 1). Then, differences observed in the TMR curves do not originate from a variation of the coupling strength.

When the absolute amplitude of the negative applied field is increased, after the switching of $\mathrm{Co}^{\text {se }}$, rotation of the magnetic grains of the hard layer proceeds continuously and a smooth decrease of the TMR signal is observed before negative saturation. In this hard layer, no growth-induced macroscopic anisotropy can be observed. Therefore, rotation occurs without an irreversible magnetization switch along an easy magnetization axis. Then, regions with the main magnetization oriented in the negative direction do not coexist with regions with the main magnetization oriented in the positive field direction. Instead, clockwise and counterclockwise rotations of neighboring magnetic grains lead to the appearance of $360^{\circ}$ domain walls parallel to the external field axis. The magnetization of each grain or of each region with an extension less than the exchange correlation length points in the direction of the negative applied field. In fact, for a given value of $H_{r e v}$, the cosine of the angle between the local magnetization and the applied field, and therefore the stray field parallel to the applied field, has the same sign all over the hard electrode volume. Then, all the regions of $\mathrm{Co}^{\text {se }}$ are submitted, along the external field axis, to the same effective field equal to $H+H_{f}^{\prime}$ since the component of the stray field perpendicular to the applied field does not contrib- 


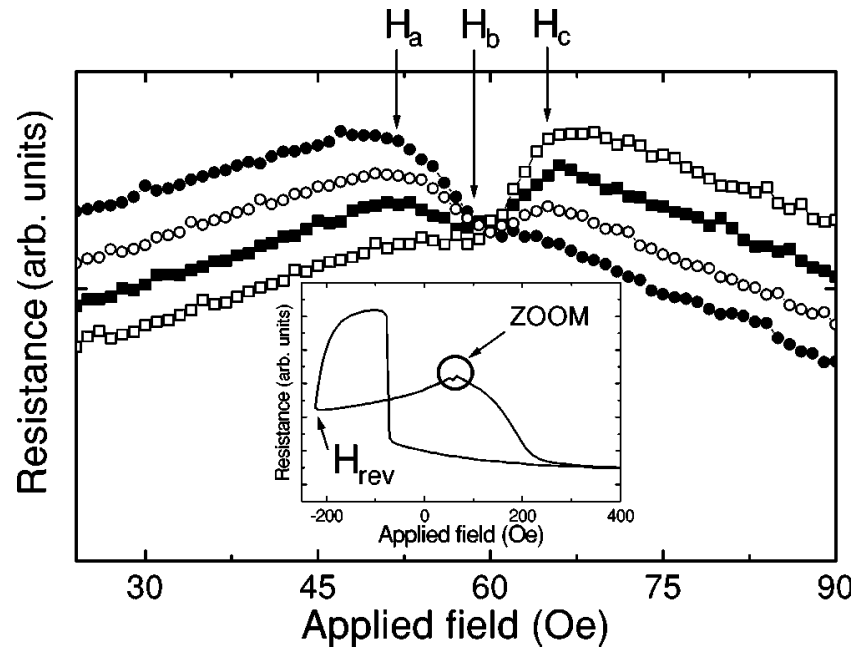

FIG. 3. Four zooms on minor tunnel magnetoresistance loops measured on a $\mathrm{Co}^{s e} / \mathrm{Al}(1.2 \mathrm{~nm}$, ox $30 \mathrm{~s}) / \mathrm{Co}^{*}$ tunnel junction with $200 \mu \mathrm{m}$ lateral size. The four different minor cycles have been obtained with decreasing value of $-H_{\text {rev }}$ for cycle $(\mathbf{O}),(\bigcirc),(\boldsymbol{\square})$, and $(\square)$. For minor cycle $(\bigcirc)$ and $(\boldsymbol{\square})$, three jumps of resistance can be observed. The inset shows a typical complete minor loop on which the zooms have been done.

ute for the reversal ( $H_{f}^{\prime}$ is the ferromagnetic field coupling between the two electrodes). The value of $H_{f}^{\prime}$ increases from a negative value to a positive value when $H_{\text {rev }}$ decreases as can be seen in Fig. 2. Indeed, $H_{f}^{\prime}$ follows a law similar to the cosine of the angle between the magnetization and the applied field at $H_{r e v}$. This cosine law is also reflected in the shape of the TMR curve after reversal of $\mathrm{Co}^{s e}$. So, since the stray field of the hard layer domain structure along the applied field direction has the same sign all over the hard layer volume, no duplication could be observed.

In this discussion, the effect of the $360^{\circ}$ domain walls has been neglected. Indeed, even if no anisotropy exists in the $\mathrm{Co}^{*}$ hard single layer, the magnetic moment at the center of the nucleated $360^{\circ}$ domain walls remains aligned along the positive saturating field direction. ${ }^{10,4}$ Therefore, on the TMR cycle, as soon as the magnetic moments of clockwise and counterclockwise rotating regions point in the negative field direction, large regions with a small magnetization component oriented along the negative field direction coexist with small regions (domain walls) with a large magnetization component oriented along the positive field direction. This situation, which runs when the resistance jump attributed to the reversal of the soft layer magnetization is low or zero, should lead to effects similar to those presented when domain structure duplication occurs. Minor TMR loops have been measured with $H_{\text {rev }}$ in a field window such that the absolute value of the resistance jump at $H_{1}$ is less than $10 \%$ of the total resistance variation on the complete TMR cycle. In Fig. 3 are reported zooms of the minor cycles in the field window of interest. Between $H_{r e v}$ and $H_{a}$, the slight increase of the TMR signal is due to a small relaxation of the grain magnetization towards the positive saturating field. Depending on the value of $H_{r e v}$, a decrease of resistance up to $H_{b}$, an increase of resistance up to $H_{c}$, and a decrease of resistance up to positive saturation can be observed [Fig. 3 $(-\bigcirc-,-\square-)]$. These three states of resistance are characteristic of domain duplication. Nevertheless, in this case, the effect is less pronounced because quite all the magnetization of the hard layer is perpendicular to the one of the soft layer due to the lack of anisotropy. So the domain duplication at localized places over the $360^{\circ}$ domain walls, the anisotropy of which is given by the initial saturating field, does not hinder the use of magnetic tunnel junctions in magnetoelectronic devices.

In summary, by using TMR measurements on a magnetic tunnel junction as a probe, we have shown the importance of the magnetic anisotropy of the hard magnetic electrode in both soft and hard layer domain structures. It induces drastic changes in each step of the reversal process which have been confirmed through a detailed analysis of the minor hysteresis loops. Moreover, through a domain structure duplication phenomena, this anisotropy is at the origin of the decrease of the TMR signal occurring in an important magnetic field range during the reversal process. Reducing this anisotropy and the magnetic interlayer coupling is a key issue to making reliable sensors. However, by looking carefully at the hysteresis loop of the samples with an isotropic hard layer, we have observed a signature of the $360^{\circ}$ domain walls.

The authors thank M. Alnot, D. Mulot, F. Mouginet, D. Pierre, E. Rafidison, and S. Robert for their technical support, and K. Ounadjela and C. Tiusan for many fruitful discussions.
${ }^{1}$ J.S. Moodera, L.R. Kinder, T.M. Wong, and R. Meservey, Phys. Rev. Lett. 74, 3273 (1995).

${ }^{2}$ L. Thomas, J. Lüning, A. Scholl, F. Nolting, S. Anders, J. Stöhr, and S.S.P. Parkin, Phys. Rev. Lett. 84, 3462 (2000).

${ }^{3}$ C.L. Platt, B. Dieny, and A.E. Berkowitz, J. Appl. Phys. 81, 5523 (1997).

${ }^{4}$ C. Tiusan, T. Dimopoulos, M. Hehn, V. Da Costa, Y. Henry, H.A.M. van den Berg, and K. Ounadjela, Phys. Rev. B 61, 580 (2000).

${ }^{5}$ L. Thomas, M.G. Samant, and S.S.P. Parkin, Phys. Rev. Lett. 84, 1816 (2000).
${ }^{6}$ M. Hehn, O. Lenoble, D. Lacour, C. Féry, M. Piécuch, C. Tiusan, and K. Ounadjela, Phys. Rev. B 61, 11643 (2000).

${ }^{7}$ M.R. McCartney, R.E. Dunin-Borkowski, M.R. Scheinfein, D.J. Smith, S. Gider, and S.S.P. Parkin, Science 286, 1337 (1999).

${ }^{8}$ C. Féry, L. Hennet, O. Lenoble, M. Piécuch, E. Snoeck, and J.F. Bobo, J. Phys.: Condens. Matter 10, 6629 (1998).

${ }^{9}$ F. Canet, O. Lenoble, and M. Piécuch, Diploma work, Université Henri Poincaré, 1996.

${ }^{10}$ K.D. Leaver, Thin Solid Films 2, 149 (1968); D.V. Berkov and N.L. Gorn, Phys. Rev. B 57, 14332 (1998). 